Manuscript prepared for Atmos. Chem. Phys.

with version 1.3 of the LTEX class copernicus.cls.

Date: 19 December 2012

\title{
Age of stratospheric air in the ERA-Interim Complementary comparison
}

\author{
M. Diallo ${ }^{1}$, B. Legras ${ }^{1}$, and A. Chédin ${ }^{2}$ \\ ${ }^{1}$ Laboratoire de Météorologie Dynamique, UMR8539, IPSL, UPMC/ENS/CNRS/Ecole Polytechnique, Paris, France \\ ${ }^{2}$ Laboratoire de Météorologie Dynamique, UMR8539, IPSL, CNRS/Ecole Polytechnique/ENS/UPMC, Palaiseau, France
}

\section{Introduction}

In this supplement, we provide additional figures in order to compare the results presented in our main article with those of Li et al. (2012) (hereafter LI12). In LI12 a free running general circulation model with comprehensive chemistry is integrated from 2000 to 2019 based on A1b scenario of IPCC and A1 ozone depleting substance scenario of WMO. The age of air is obtained from the pulse response method applied to all months of 2000 with pulses released in the boundary layer. The authors claim that the GEOSCCM model "has the best performance in mean age among all the CCM that participated in the CCMVal-2."

We have performed a modified analysis using potential temperature as vertical coordinate and drawn figures which can be directly compared with LI12. The potential temperature discretization is obtained by splitting the interval [300K $1500 \mathrm{~K}]$ into 22 sub-intervals which are regularly distributed in the log scale. The latitude discretization is the same as in the main article with 2-degree bins except near the poles where latitudes are grouped into enlarged latitude bins $\left(69^{\circ}-\right.$ $73^{\circ}, 73^{\circ}-77^{\circ}, 77^{\circ}-81^{\circ}, 81^{\circ}-90^{\circ}$ ) to improve the statistics. The ages are then grouped per months over the 22 years of the record between 1989 and 2010.

\section{Mean age seasonal cycle}

Figure 1 shows the mean monthly ages and monthly anomalies over the same months and in the same axis as Fig. 5 of LI12. The similarity of the patterns between the two figures is striking even in the smallest details. During January and March in the Northern hemisphere, and during July and September in the southern hemisphere, the descent of old air over the winter pole induces a positive age anomaly. By compensation, a negative anomaly occurs at the summer pole.

Correspondence to: mdiallo@lmd.ens.fr
The patterns of the anomalies as well as the strengthening of the meridional age gradient in the southern hemisphere during winter are very similar in LI12 and in our work. We find a less intense strength during November in our study but this is because the polar vortex lasted very late during the austral 2000 spring. Other maxima and minima at $30^{\circ} \mathrm{S}$ and $30^{\circ} \mathrm{N}$ above $450 \mathrm{~K}$ are also similar to those found in LI12.

The differences arise as a general shift towards older ages above $500 \mathrm{~K}$ in our study which reaches $2 \mathrm{yr}$ in the winter polar region. A positive shift of the order of a few months is expected because LI12 calculate the age of air with respect to a release in the lower troposphere below $800 \mathrm{hPa}$ while we calculate it with respect to the crossing of the tropopause. However differences of the order of $2 \mathrm{yr}$ cannot be explained that way.

Figure 2 shows the annual cycle after removal of the contributions from all the parcels which have reached altitudes with $\theta>1800 \mathrm{~K}$, that is essentially $p<2 \mathrm{hPa}$. The same filter was also applied by Schoeberl and Dessler (2011). The patterns are still the same as in Fig. 1 but the amplitudes are now reduced and much more in agreement with Fig. 5 of LI12. This ad hoc filtering demonstrates the significant contribution of the upper stratospheric and mesospheric circulations to the tail of the age spectrum and to the mean age. It also suggests, in comparison with LI12, either that parcels reside too long at high altitude in the ERA-Interim, or that the mean tropical upwelling is too small. This latter hypothesis is not supported by the diagnostics of residual circulation shown by Seviour et al. (2011).

\section{Seasonal variation of the age spectrum}

Figures 3-5 show the seasonal cycle of the age spectrum $f(\tau)$ at the same altitudes and latitudes as Figs 8-10 of LI12, except at the lowest level $360 \mathrm{~K}$ where the latitudes $20^{\circ} \mathrm{N}$ and $20^{\circ} \mathrm{S}$ have been replaced by $36^{\circ} \mathrm{N}$ and $36^{\circ} \mathrm{S}$. Because we 
are counting ages for stratospheric parcels from the crossing of the tropopause, it is not possible to define ages at locations below the tropopause unlike LI12 who launch parcels in the lower troposphere. In addition to the modal age, corresponding to the peak of the spectrum, three mean ages are shown on the figures:

- the integral mean age over the truncated spectrum which is calculated as

$$
<\tau>=\frac{\sum_{i=1}^{K} n_{i} t_{i}}{\sum_{i=1}^{K} n_{i}}
$$

with the notations of Sec. 2.3 of the main text,

- the mean corrected age calculated with Eq. (3) of the main text over the $N$ released parcels

- the mean truncated age calculated over the subset of $N^{\prime}$ released parcels which have always remained below the level $\theta=1800 \mathrm{~K}$.

At the lowest level, for $\theta=360 \mathrm{~K}$, the two left panels of Fig. 3 show a modal age locked near zero because of the proximity of the tropopause while the modal ages of LI12 at the same level indicates the transport time from the lower troposphere. The mean ages at $46^{\circ} \mathrm{N}$ and $46^{\circ} \mathrm{S}$, however, exhibit variations of the same shape and amplitude as in Fig. 8 of LI12. At polar latitudes, the modal age exhibits the same behaviour as in LI12 with an offset of a few months. The mean corrected age agrees perfectly with LI12 at $79^{\circ} \mathrm{N}$ but is slightly older at $79^{\circ} \mathrm{S}$.

At the mid-level, for $\theta=420 \mathrm{~K}$, the modal shift is still visible at all latitudes and the mean corrected age agrees well with the mean age of LI12 in the northen hemishere while it is older in the southern hemisphere, by about one yr at the pole. The contrast between winter and summer at the southern pole is very similar in both calculations. The annual propagating modulation of the spectrum is already clearly visible at this level.

At the upper level, for $\theta=550 \mathrm{~K}$, the modulation is present at tropical latitudes and dominates at mid and high latitudes over a fairly flat background (see Fig. 7 of the main article). The mean corrected age is now always larger than the mean LI12 age but the mean truncated age is very similar. The sharp cutoff at small ages associated with the isolating effect of the polar vortex is in particularly good agreement. The patterns and the amplitude of the modulation are very similar with LI12 at low and mid-latitudes. A significant difference is that the phase of the oscillations is shifted by about six months in the polar stratosphere.

A remarkable property is that the oscillations are all in phase at any altitude and latitude, and that the crest occurs for an integer age during winter. This is consistent with the interpretation of Reithmeier et al. (2008) that they are entirely due to the modulation of the tropical upwelling which is maximum during winter.
Notice that the patterns of the normalized spectrum do not change when parcels which have gone above $1800 \mathrm{~K}$ are removed, consistently with the similarity between Fig 1 and 2 .

\section{Conclusions}

We have compared the age of stratospheric air in the ERAInterim and in a GEOSCCM experiment. It is found that the age distribution of the normalized air are strikingly similar, in the mean, the seasonal variability and the age spectra. There are significant differences in the amplitude, the ERA-Interim generating older ages by up to $2 \mathrm{yr}$ at high latitude. Since the methods used to estimate the ages are very different, one cannot a priori rule out a computational bias. However these differences are larger than those between the kinematic and diabatic ages in the ERA-Interim. It is noticeable that the decay rate of the age spectrum in GEOSCCM is $0.36 \mathrm{yr}^{-1}$, that is almost twice the decay rate of the ERAInterim which is $0.20 \mathrm{yr}^{-1}$. Since the mean upwelling flux of the ERA-Interim is in the same range as that of CCMs (Seviour et al., 2011), its is thus likely that parcels recirculate within the stratosphere in the ERA-Interim and our analysis with truncated trajectories indicate that this circulation occurs above $1800 \mathrm{~K}$. The top lid of the ERA-Interim is located at $0.1 \mathrm{hPa}$ while the top lid of GEOSCCM is at $0.001 \mathrm{hPa}$. Further studies are needed to confirm the effect of mesospheric circulation on the mean age of air.

\section{References}

Li, F., Waugh, D. W., Douglass, A. R., Newman, P. A., Pawson, S., Stolarski, R. S., Strahan, S. E., and Nielsen, J. E.: Seasonal variations of stratospheric age spectra in the Goddard Earth Observing System Chemistry Climate Model (GEOSCCM), J. Geophys. Res., 117, D05 134, doi:10.1029/2011JD016877, 2012.

Reithmeier, C., Sausen, R., and Grewe, V.: Investigating lower stratospheric model transport: Lagrangian calculations of mean age and age spectra in the GCM ECHAM4, Climate Dynamics, 30, 225-238, doi:10.1007/s00382-007-0294-1, 2008.

Schoeberl, M. R. and Dessler, A. E.: Dehydration of the stratosphere, Atmos. Chem. Phys., 11, 8433-8446, doi:10.5194/ acp-11-8433-2011, 2011.

Seviour, W. J. M., Butchart, N., and Hardiman, S. C.: The BrewerDobson circulation inferred from ERA-Interim, Q. J. R. Meteorol. Soc., 138, 878-888, doi:10.1002/qj.966, 2011. 

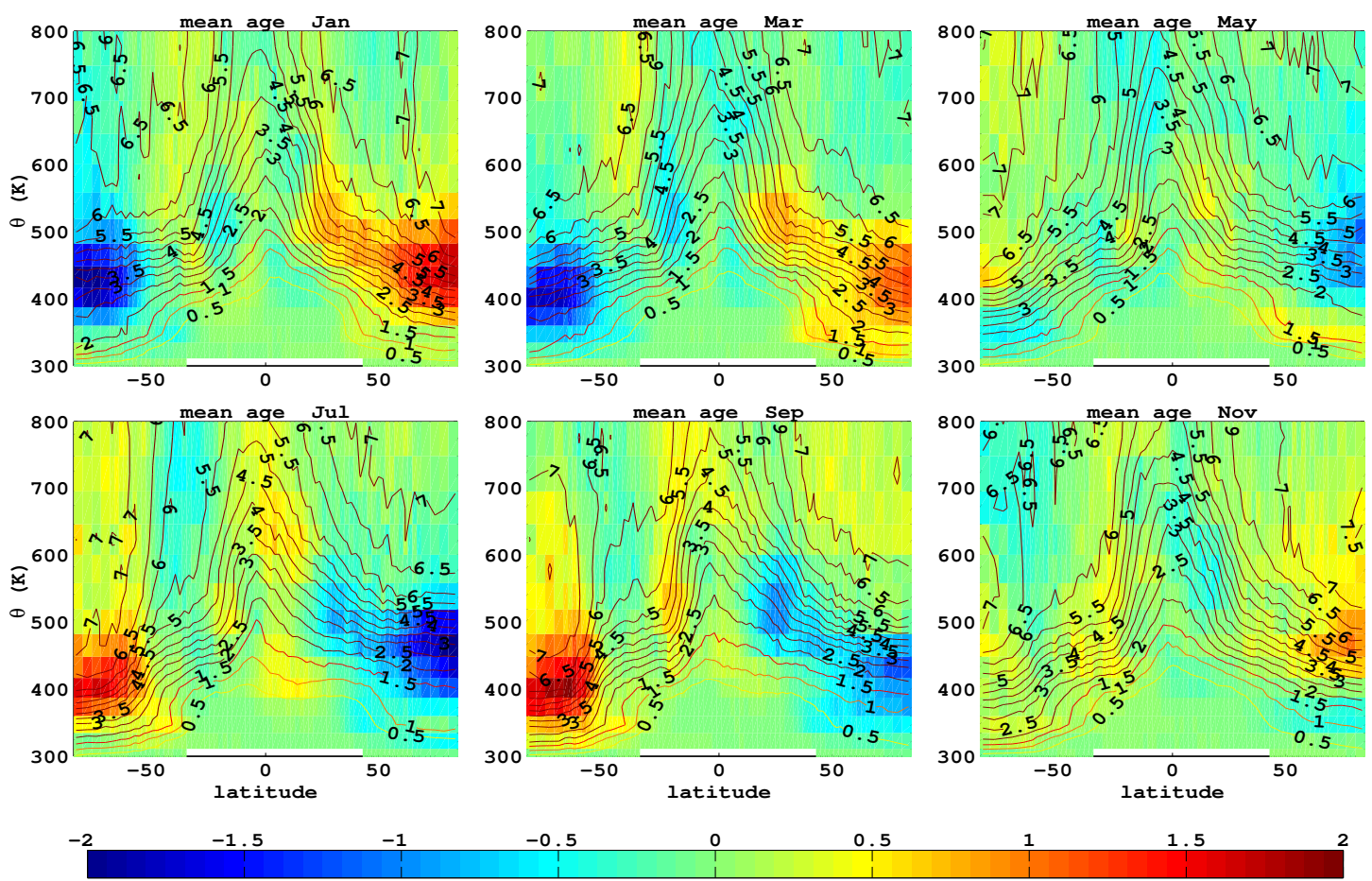

Fig. 1. Seasonal cycle of the mean age shown as latitude-altitude crossections for January, March, May, July, September and November. The monthly mean age is shown as contours and the monthly anomaly is shown by colors.
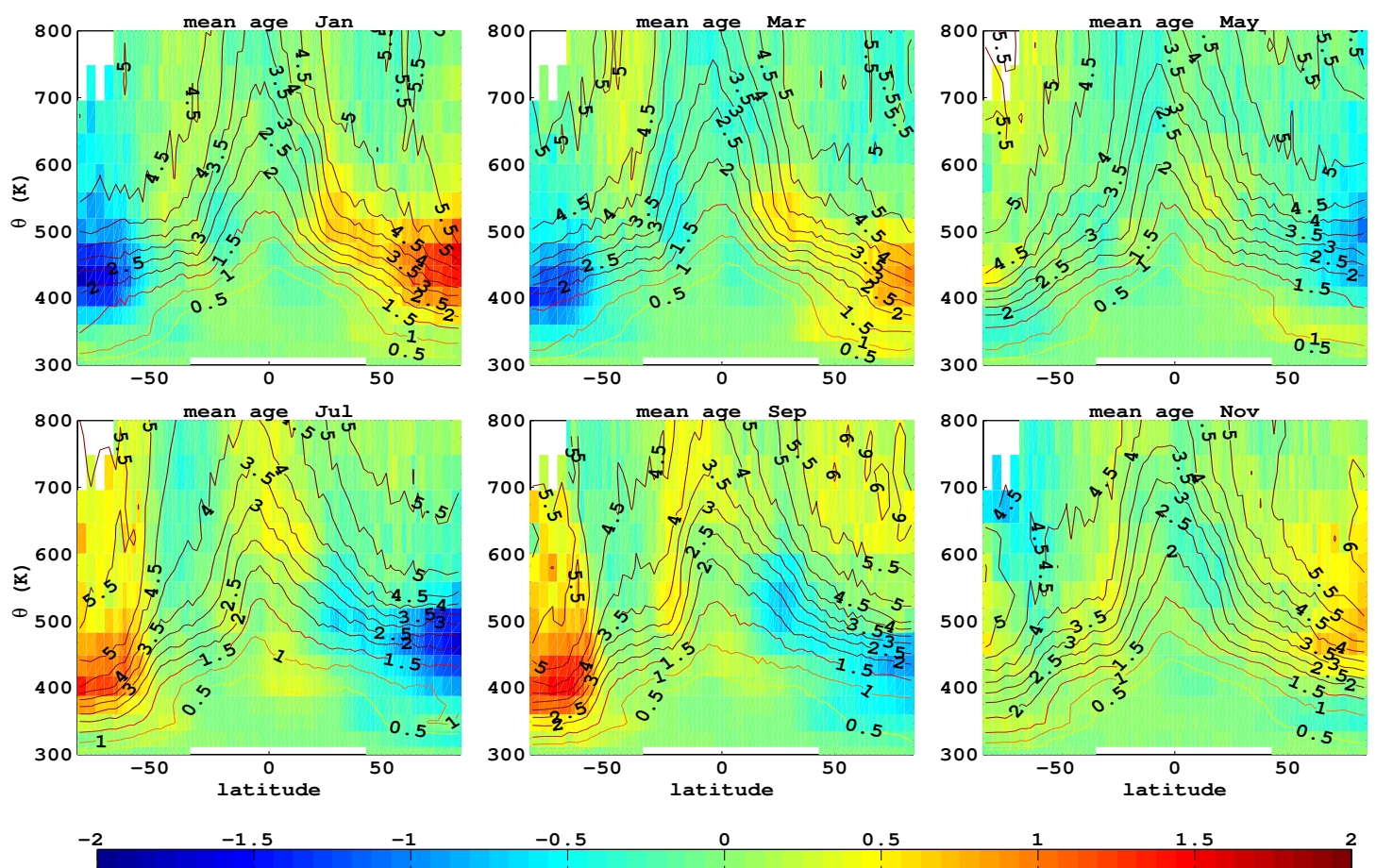

Fig. 2. Same as Fig. 1 but excluding all particles which have reached potential temperatures larger than $1800 \mathrm{~K}$. 

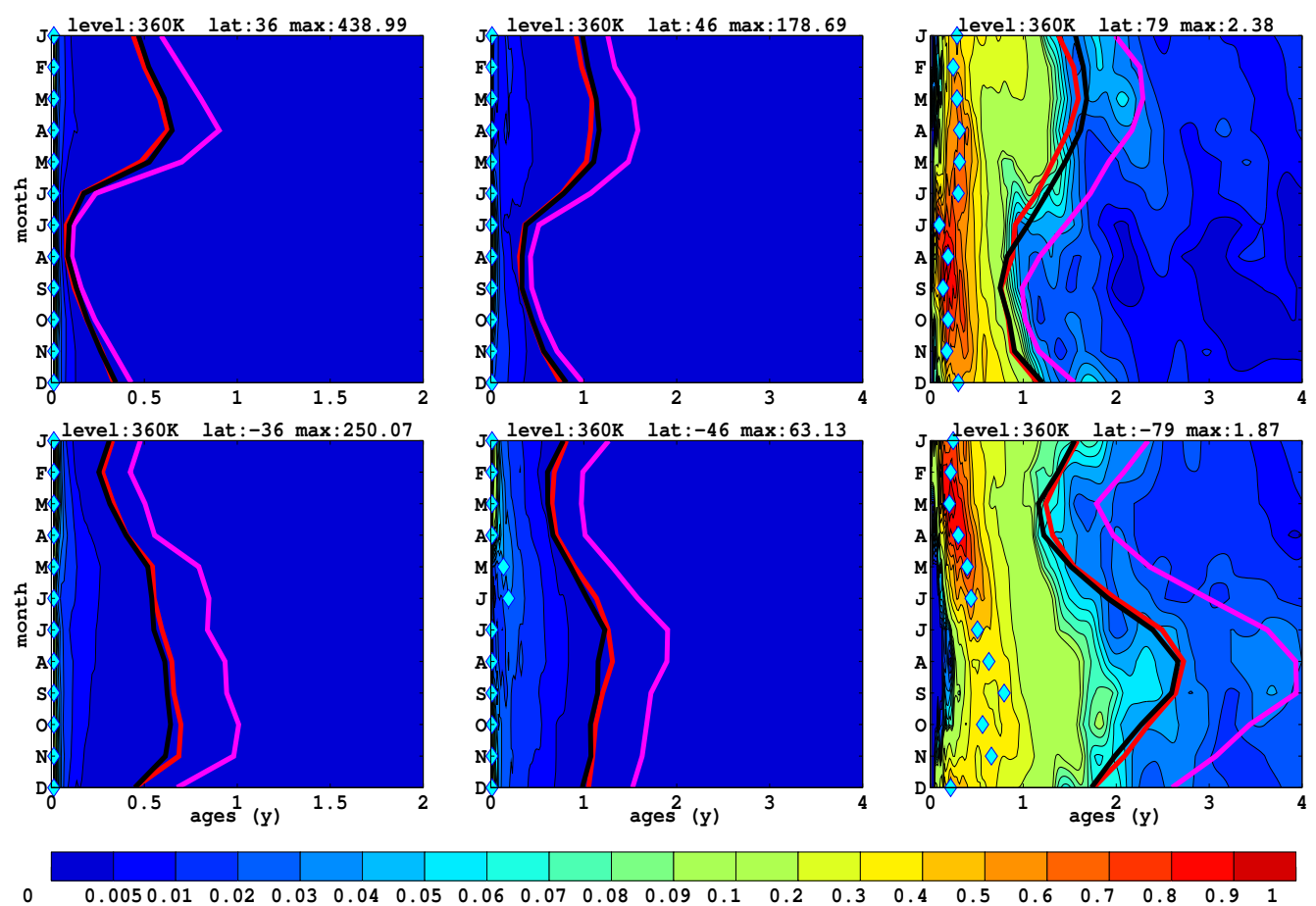

Fig. 3. Seasonal cycle of the age spectra at $36^{\circ}, 46^{\circ}$ and $80^{\circ}$ latitude north and south and at level $\theta=360 \mathrm{~K}$. The age density is normalized by its maximum value in each panel. Dots: modal age. Red line: mean age obtained by integration over the age spectrum up to 10 yr. Magenta line: mean corrected age as defined in Sec. 2.3 of the main text. Black line: mean corrected age obtained with particles which have always remained below $\theta=1800 \mathrm{~K}$. 

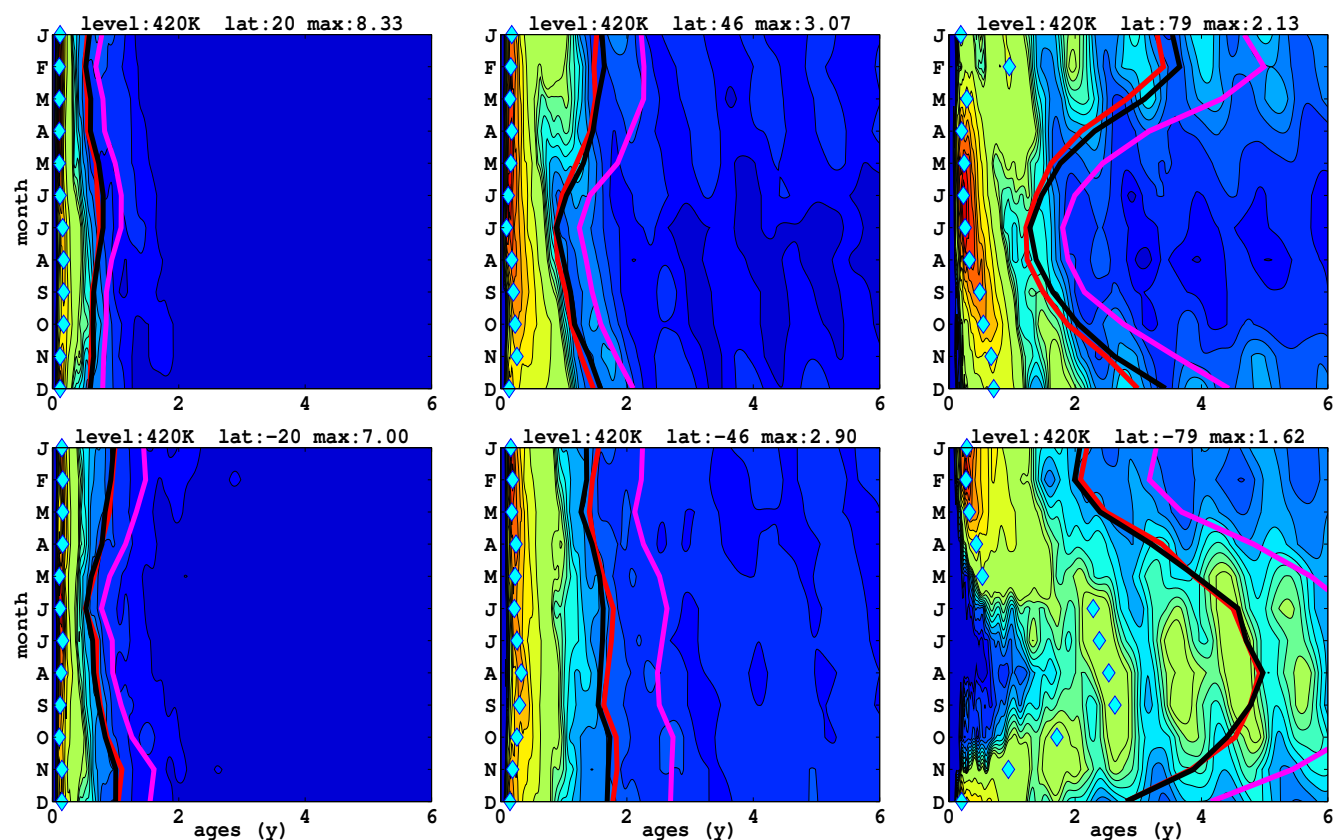

0

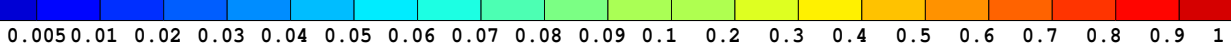

Fig. 4. Same as Figure 4 but for $\theta=420 \mathrm{~K}$ and at $20^{\circ}$ north and south in the two left panels, as in LI12.
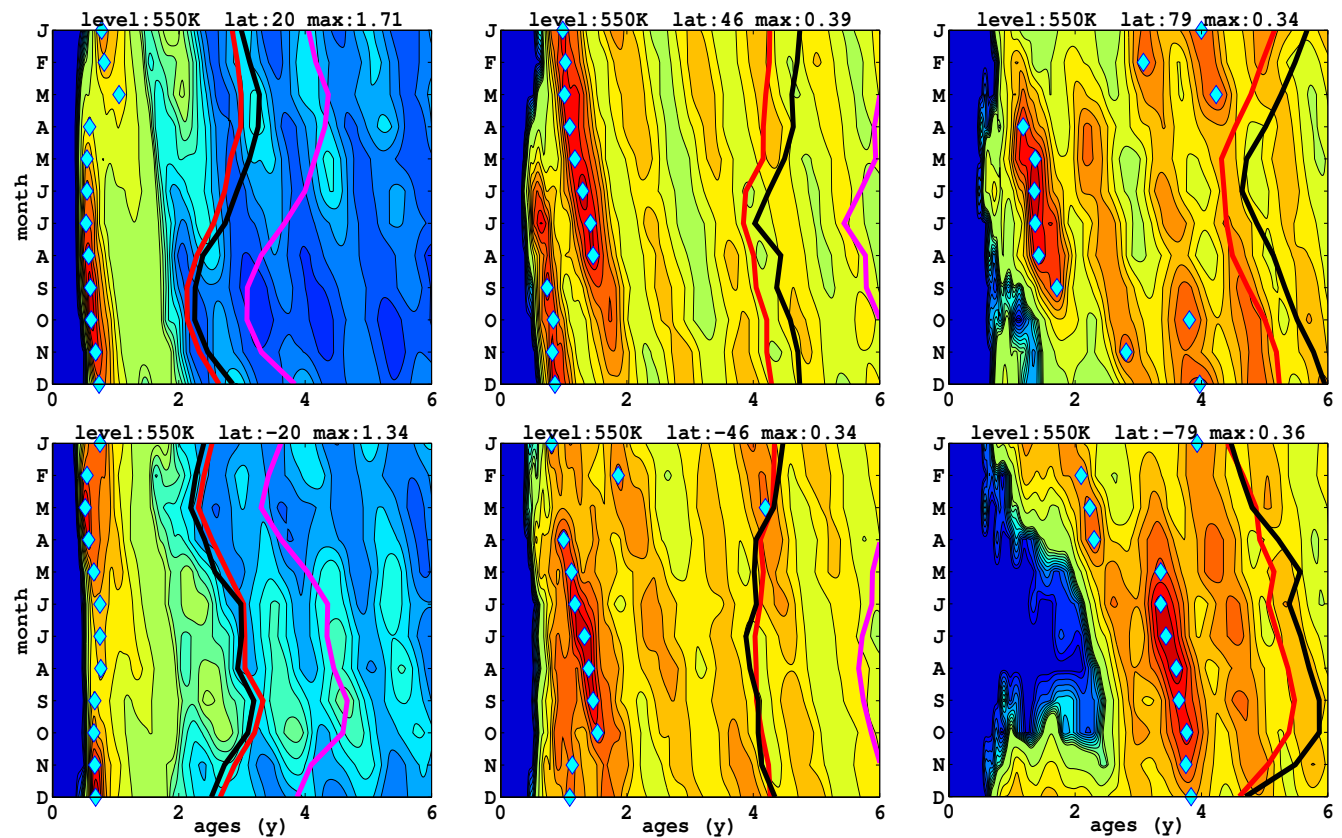

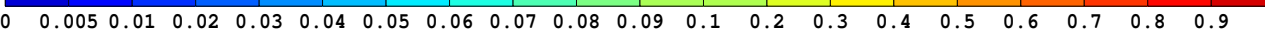

Fig. 5. Same as Figure 4 but for $\theta=550 \mathrm{~K}$. 